\title{
DEUTSCHER SPORTBUND
}

\section{Hermann-Altrock-Stipendium Ausschreibung 2003}

\section{Ausführungsbestimmungen:}

1. In Würdigung der Verdienste von Professor Dr. Hermann ALTROcK (1887-1980) um Aufbau und Gestaltung des Studiums der Leibeserziehung sowie um den organisierten Sport in Deutschland schreibt der Deutsche Sportbund zum 36. Mal das Hermann-Altrock-Stipendium aus.

2. Das Stipendium soll Promovendinnen/Promovenden in der Sportwissenschaft Beginn oder Fertigstellung einer thematisch wichtigen Dissertation ermöglichen. Das Stipendium hat ein Volumen von $6000 €$ und kann auf mehrere Promovendinnen/Promovenden aufgeteilt werden.

3. Vorschläge für die Verleihung sind durch die sportwissenschaftlichen Hochschuleinrichtungen oder Promovendinnen/Promovenden bis zum 15. August 2003 an den Deutschen Sportbund, Stabsstelle „Grundsatzfragen, Wissenschaft und Gesellschaft“, Postfach, 60528 Frankfurt am Main, zu senden.

4. Einzureichen sind

- Referenzen von zwei Hochschullehrerinnen/Hochschullehrern

- Lebenslauf der Bewerberin/des Bewerbers mit Lichtbild

- Zeugnis über den Abschluss des sportwissenschaftlichen Studiums

- Angebot über Thema, Aufbau und Stand des Promotionsvorhabens sowie Darlegung der beabsichtigten Verwendung des Stipendiums

- Bericht über die Mitarbeit im Studium, insbesondere in Seminaren und an Forschungsvorhaben

- Bericht über ehrenamtliche, neben- oder hauptberufliche Tätigkeiten im organisierten Sport

5. Die Entscheidung über die Bewerbungen trifft der Deutsche Sportbund.

6. Entscheidung und Verteilung sind für das IV. Quartal des Jahres 2003 vorgesehen.

Frankfurt am Main, im März 2003 